\title{
EAR SEGMETATION USING TOPOGRAPHIC LABELS
}

\author{
Milad Lankarany, Alireza Ahmadyfard \\ Department of Electrical Engineering and Robotics, Shahrood University of Technology, Shahrood, Iran \\ milad.lankarany@gmail.com
}

Keywords: $\quad$ Ear Biometrics, Ear Segmentation, Topographic Features

\begin{abstract}
Ear segmentation is considered as the first step of all ear biometrics systems while the objective in separating the ear from its surrounding backgrounds is to improve the capability of automatic systems used for ear recognition. To meet this objective in the context of ear biometrics a new automatic algorithm based on topographic labels is presented here. The proposed algorithm contains four stages. First we extract topographic labels from the ear image. Then using the map of regions for three topographic labels namely, ridge, convex hill and convex saddle hill we build a composed set of labels. The thresholding on this labelled image provides a connected component with the maximum number of pixels which represents the outer boundary of the ear. As well as addressing faster implementation and brightness insensitivity, the technique is also validated by performing completely successful ear segmentation tested on "USTB" database which contains 308 profile view images of the ear and its surrounding backgrounds.
\end{abstract}

\section{INTRODUCTION}

Ear images can be acquired in a similar manner to face images and a number of researchers have suggested that the human ear is unique enough to each individual to allow practical use as a biometric. Bhanu and Chen [2003] presented a 3D ear recognition method using a local surface shape descriptor. Twenty range images from 10 individuals are used in the experiments and a 100 percent recognition rate is reported. Chen and Bhanu [2005] used a two-step ICP algorithm on a data set of 30 subjects with $3 \mathrm{D}$ ear images. They reported that this method yielded two incorrect matches out of 30 people. In these two works, the ears are manually extracted from profile images. They also presented an ear detection method in [Bhanu, 2004]. In the offline step, they built an ear model template from each of 20 subjects using the average histogram of the shape index. In the online step, first, they used step edge detection and thresholding to find the sharp edge around the ear boundary and then applied dilation on the edge image and connected component labelling to search for ear region candidates. Each potential ear region is a rectangular box, and it grows in four directions to find the minimum distance to the model template. The region with minimum distance to the model template is the ear region. They get 91.5 percent correct detection with a 2.5 percent false alarm rate. No recognition results are reported based on this detection method.

Hurley et al developed a novel feature extraction technique using force field transformation. Each image is represented by a compact characteristic vector which is invariant to initialization, scale, rotation, and noise. The experiment displays the robustness of the technique to extract the $2 \mathrm{D}$ ear. The data set comes from the XM2VTS face image database. Choras introduces an ear recognition method based on geometric feature extraction from $2 \mathrm{D}$ images of the ear. The geometric features are computed from the edge detected intensity image. They claim that error-free recognition is obtained on "easy" images from their database. The "easy" images are images of high quality with no earring and hair covering and without illumination changes. No detailed experimental setup is reported.

There are a number of algorithms based on force field transforms to deal with segmentation. Luo et al describe the use of Vector Potential to extract corners by treating the "Canny" edge map of an image as a current density. Ahuja used a novel force field segmentation technique where pixels of similar intensity were detected by assigning inter-pixel forces inversely proportional to the grey level difference. Ahuja and Chuang used a potential field 
model to extend the medial axis transform. $\mathrm{Xu}$ and Prince extended the active contour model by replacing the external local force with a force field derived from the image edge map.

Yan et al present the first fully automated system for ear biometrics using 3D shape. There are two major parts of the system: automatic ear region segmentation and 3D ear shape matching. Starting with the multimodal $3 \mathrm{D}+2 \mathrm{D}$ image acquired in a profile view, the system automatically finds the ear pit by using skin detection, curvature estimation, and surface segmentation and classification. After the ear pit is detected, an active contour algorithm using both colour and depth information is applied to outline the visible ear region.

Except the Yan's method that automatically approached ear-segmentation using 3-D information of the profile images, the works based on edge detection algorithms present automated approaches for ear-segmentation using only 2-D images of the ear. In this case, the edge image of the ear and surroundings is first obtained then distinctive contours are recognized. It is experimentally concluded that the contour which includes the maximum number of pixels, points out to the edge of outer ear. Therefore the ear boundary can be found easily according to that contour. However, this technique suffers two main problems. First problem is caused by the dependence of edge detection algorithms to thresholding value. Second one arises from the discontinuity of contours obtained from the edge detector. In some cases, several pixels of a same contour, which is detectable using human's eyes, are disconnected therefore; the separated contours are recognized wrongly. This effect is illustrated in figure 1 .

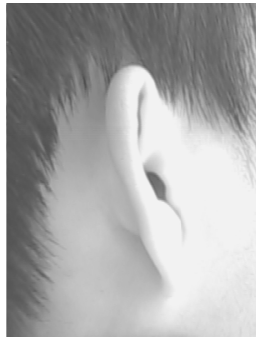

(a)

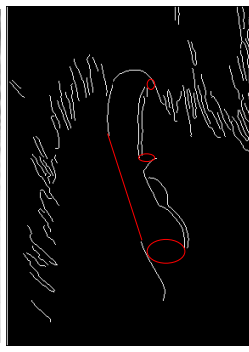

(b)

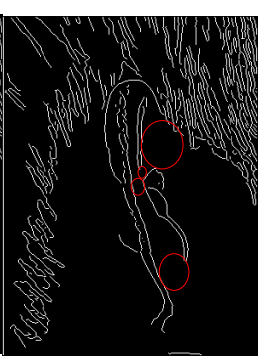

(c)
Figure1: (a). A profile image from USTB database, (b). The edge image using "canny" operator with threshold 0.2

(c). The edge image with threshold 0.1 (the red line and ellipse show the discontinuity of contours).

These problems motivated us to use a method leading to obtain more reliable regions (instead of edge contours) free from threshold selection.
Providing this purpose, we used topographic map of ear image and label it. Each pixel in ear image is described by one of the twelve topographic labels. No predefined threshold is needed to label the image in this manner. On the other hand, more geometric properties of the ear image are yielded. Three labels, namely, ridge, convex hill and convex saddle hill which are selected experimentally, are combined in order to extract the ear outline. More consideration in outer ear shape shows that this part of ear is fully described by the convex hill and convex saddle hill labels. Moreover the ridge label in the ear image is similar to the image edges, but the extraction of the ridge label is independent of threshold value. Figure 2 shows the each of those labels extracted from a test ear. It is experimentally concluded that the connected component obtained from the combination of topographic labels consisting of the maximum number of pixels matches to the image of outer ear. As a result the boundary of this region demonstrates the ear in an ear image.

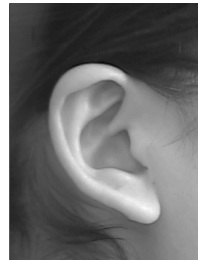

(A)

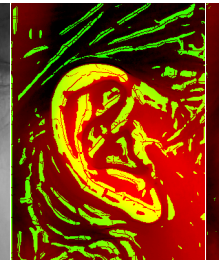

(B)

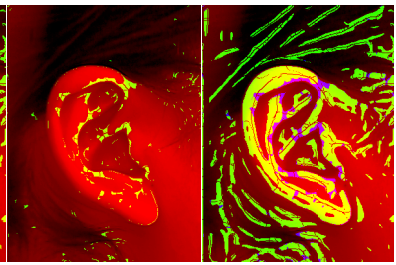

(C)

(D)
Figure 2: (A). Selected image from USTB database, (B). Green part shows the convex hill label, (C). Green part indicates the convex sadlle hill, (D). Green part shows convex hill and blue part shows the convex saddle hill label, all extracted from selected ear image.

\section{TOPOGRAPHIC LABELS}

Using topographic models for representing images has been reported in computer vision literature [M.Haralick, 1983 and J.Wang, 2007]. This method is classified in appearance based category. The main advantage of using topographic features for representation respect to intensity is its robustness to lighting condition [L.Wang, 1993].

Consider a grey scale ear image as a surface in $3 \mathrm{D}$ space where $x$ and $y$ axes are along image dimensions. The value of surface at pixel $(x, y)$ is the pixel intensity $f(x, y)$. Depending on the topographic property of surface at each pixel one of twelve topographic labels in Figure 3 is assigned to the each pixel. In order to label intensity image based on topographic property let us consider the input image 
as a continues function $f(x, y)$. The topographic label at each pixel of image is determined using first and second order derivatives on surface $f$.

Considering the Hessian matrix of this function as follows:

$$
H=\left[\begin{array}{ll}
\frac{\partial^{2} f(x, y)}{\partial x^{2}} & \frac{\partial^{2} f(x, y)}{\partial y} \\
\frac{\partial^{2} f(x, y)}{\partial y} & \frac{\partial^{2} f(x, y)}{\partial y^{2}}
\end{array}\right]=\left[\begin{array}{ll}
f^{(2,0)}(x, y) & f^{(1,1)}(x, y) \\
f^{(1,1)}(x, y) & f^{(0,2)}(x, y)
\end{array}\right]
$$

After applying eignvalue decomposition to the Hessian matrix we have:

$$
\begin{aligned}
& H=U D U^{T}= \\
& {\left[\begin{array}{ll}
u_{1} & u_{2}
\end{array}\right] \cdot \operatorname{diag}\left(\lambda_{1} \quad \lambda_{2}\right) \cdot\left[\begin{array}{ll}
u_{1} & u_{2}
\end{array}\right]^{T}}
\end{aligned}
$$

Where $\vec{u}_{1}, \vec{u}_{2}$ point out to the eigenvectors of $\mathrm{H}$ and $\lambda_{1}, \lambda_{2}$ are the eigenvalues of the Hessian matrix. Also $\vec{\nabla} f, \vec{\nabla} f$ correspond to the derivative vector of function $f$ (the surface intensity) and its magnitude, respectively, should be computed.

$$
\begin{aligned}
\vec{\nabla} f & =\left(\frac{\partial f(x, y)}{\partial x}, \frac{\partial f(x, y)}{\partial y}\right) \\
\|\vec{\nabla} f\| & =\sqrt{\left(\frac{\partial f(x, y)}{\partial x}\right)^{2}+\left(\frac{\partial f(x, y)}{\partial y}\right)^{2}}
\end{aligned}
$$

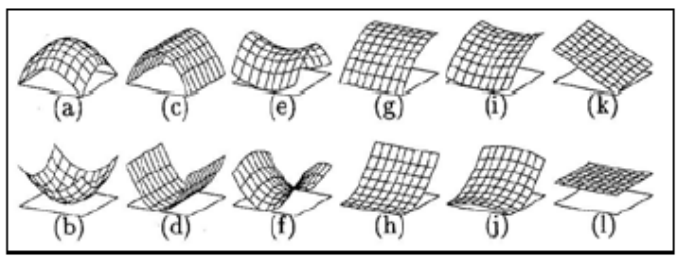

Figure 3: Topographic labels (a) peak (b) pit (c) ridge (d) ravine (e) ridge saddle (f) ravine saddle (g) convex hill (h) concave hill (i) convex saddle hill (j) concave saddle hill (k) slop hill and (l) flat

In this part we only consider the labels used in this paper. These labels are ridge, convex hill, convex saddle hill. For further details on determining other topographic labels one can refer to [L.Wang, 1993]. It is worth to note that image noise can cause an undesired result of topographic labelling. As shown by Wang et al [1993] a smoothing filter before topographic labelling provide more acceptable result. For this purpose we filter input image using a Gaussian kernel before the labelling. The filter parameters should be selected based on the size of interest pattern (ear) and the level of input noise.

Ridge: this label assigns to pixel $(\mathrm{x}, \mathrm{y})$ if one of the following criteria is satisfied.

$$
\begin{array}{ll}
\|\vec{\nabla} f(x, y)\|=0, & \lambda_{1}>0, \lambda_{2}=0 \\
\|\vec{\nabla} f(x, y)\| \neq 0, & \lambda_{1}>0, \vec{\nabla} f \cdot \vec{u}_{1}=0 \\
\|\vec{\nabla} f(x, y)\|=0, & \lambda_{2}>0, \vec{\nabla} f \cdot \vec{u}_{2}=0
\end{array}
$$

Convex hill: this label assigns to pixel $(\mathrm{x}, \mathrm{y})$ if one of the following criteria is satisfied.

$$
\begin{array}{ll}
\|\vec{\nabla} f(x, y)\| \neq 0, & \lambda_{1}>0, \lambda_{2}>0 \\
\|\vec{\nabla} f(x, y)\| \neq 0, & \lambda_{1}>0, \lambda_{2}=0
\end{array}
$$

Convex saddle hill: this label assigns to pixel $(\mathrm{x}, \mathrm{y})$ if the following criterion is satisfied.

$$
\|\vec{\nabla} f(x, y)\| \neq 0, \quad \lambda_{1}>0, \lambda_{2}<0
$$

Figure 4 (up) is plotted to indicate 3 mentioned labels extracted of figure 2.A.

\section{EAR SEGMENTATION}

A. Label Extraction: according to last section, 3 labels, namely, ridge, convex hill and convex saddle hill are extracted from each profile image. Figure 4 (down) shows the combination of these labels. B. Thresholding: the image approached by combining those three labels is binary (intensities are "0" or " 1 "). The pixels whose intensities are "1" are replaced by their original intensity values from the profile image. These values are scaled in the range of grey level (" 0 " to " 255 "). The achieved image is enhanced by using histogram equalization. At last by defining threshold value as " 150 " (in grey level) the pixels whose intensity values are lower than that threshold are mapped to " 0 " and the other pixels give " 1 " as their intensity value. Figure 5 demonstrates the image yielded by using this step. It should be noted that this step is not an essential part of our algorithm and the proposed algorithm operates accurately without using this step. But a considerable reduction in computation complexity is achieved by using this step. As compared with figure 4 (down), the excessive contours around the ear can be easily omitted using this step. C. Finding the region of outer ear: as mentioned in introduction, it is experimentally concluded that the contour which contains the maximum number of pixels covers the region of the outer ear. Figure 6 shows this contour which is extracted of figure 5 . For this purpose (finding the contours of a binary image) we use "bwlabel" a command of MATLAB. 


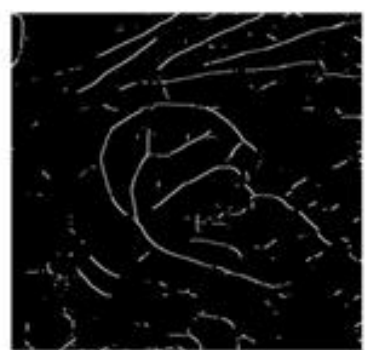

ridge

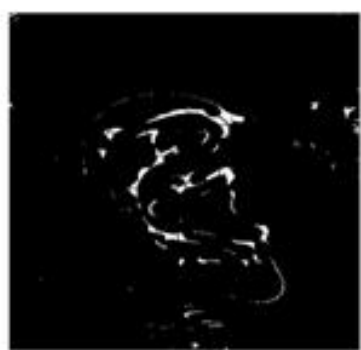

Convex saddle hill

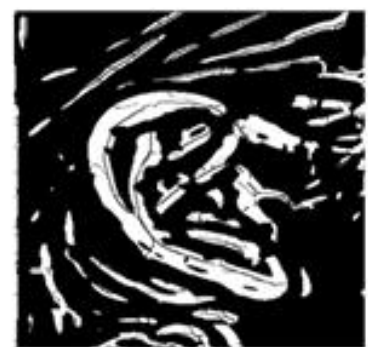

Convex hill

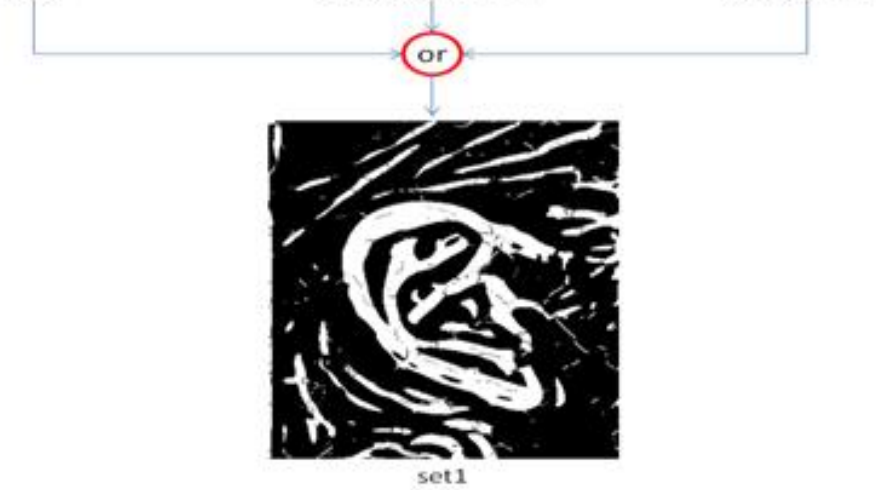

Figure4: Up. Three topographic labels obtained for profile image shown in figure 2.A

Down. The combination of those labels.

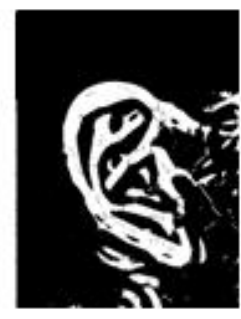

Fig5: The combination of three mentioned topographic labels after thresholding

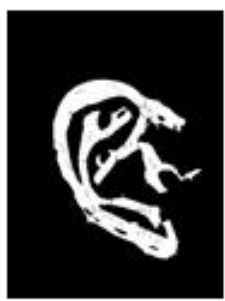

Fig6: the contour includes the maximum number of pixels

D. Two landmark points: because the features for a recognition algorithm should be invariant to translation and scale change, the normalization step is performed. The normalization process is done with respect to scale and rotation. This is done by finding a two point land mark (figure 7) in every contour approached in step C. These two points can be easily determined by finding the pixels assigned to maximum and minimum values of coordinate axis in the vertical direction.

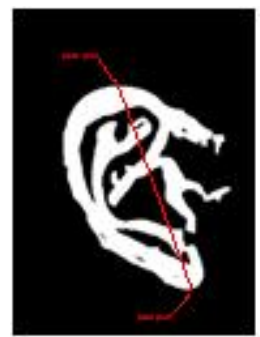

Figure7: two landmark points, which correspond to maximum and minimum values of vertical axis, are identified in the contour approached in step $\mathrm{C}$ followed by dilating.

E. Scaling and Rotation: After detecting those points, the profile image is transformed with respect to those landmarks to a new location. This transformation will result in getting the landmark points in all images to have the same distance between them and that orientation of the line connecting the two landmark points will be vertical in the image. Figure 8 demonstrates the result of this normalization applied to profile image shown in figure 2.A. 


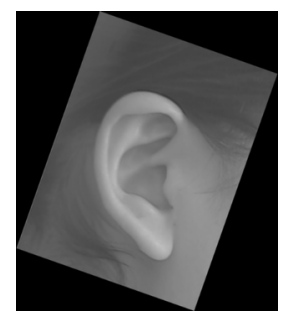

Figure8: the result of normalization of profile image with respect to rotation.

F. foure landmark points: step $E$ (normalization) is first applied to the contour yielded in step $\mathrm{C}$ then, a 4 point landmark, which are shown in figure 9.A, are selected by finding the maximum and minimum values of coordinate axises in both vertical and horizontal directions. A rectangular window separates the region of normalized ear from it's surroundings based on points obtained in step F. Figure 9.B shows this window.

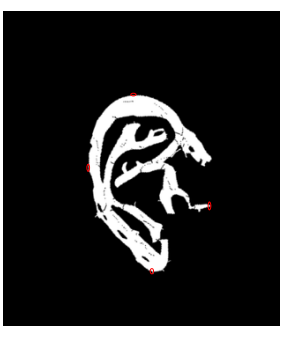

(A)

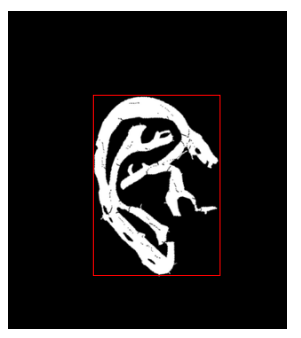

(B)
Figure 9: (A). Four land mark points founded in the normalized contour which approached by applying step $\mathrm{E}$ to the contour obtained in step C, (B). The window which is drawn based on the landmark points.

At last by applying this window to the normalized profile image approached in step E, the segmented ear will be obtained.

\subsection{Segmentation Results}

Our algorithm achieves fully successful ear segmentation on USTB database which includes 77 subject dataset, 4 images for each subject. Some profile images selected of our used database are shown in figure 10.A. Ear segmentation based on proposed algorithm achieves 100 percent true segmentation while applying edge detection algorithm according to "Canny" method, yielded
67.2 percent true segmentation. Both methods are tested on the USTB database. Wrong segmentation corresponds to incomplete separation of ear from surroundings. Figure 10.B demonstrates some segmented ears, which are separated from profile images shown in figure 10.A, approached by our proposed algorithm.

\section{CONCLUSION}

We have presented an automatic ear segmentation approach using 2D images. The automatic ear extraction algorithm can separate the ear from surrounding area including hair. The proposed method uses topographic labels to obtain a reliable region representing outer ear. The experimental results demonstrate the power of our automatic ear extraction algorithm when it is compared with edge detection based techniques. The proposed system successfully segments all ear images in USTB database.

\section{REFERENCES}

Ping Yan and Kevin W.Bowyer "Biometric Recognition Using 3-D Ear Shape”, IEEE Trans. PAMI, VOL. 29, NO. 8, 2007

David J. Hurley, Mark S. Nixon, John N. Carter "Force Field Feature Extraction for Ear Biometrics", Computer Vision and Image Understanding ELSEVIER.

B. Bhanu and H. Chen, "Human Ear Recognition in 3D," Proc.Workshop Multimodal User Authentication, pp. 91-98, 2003.

D. Hurley, M. Nixon, and J. Carter, "Force Field Energy Functionals for Image Feature Extraction," Image and Vision Computing J., vol. 20, pp. 429-432, 2002.

H. Chen and B. Bhanu, "Human Ear Detection from Side Face Range Images," Proc. Int'1 Conf. Image Processing, pp. 574-577, 2004.

H. Chen and B. Bhanu, "Contour Matching for 3D Ear Recognition," Proc. Seventh IEEE Workshop Application of Computer Vision,pp. 123-128, 2005.

K. Messer, J. Matas, J. Kittler, J. Luettin, and G. Maitre, "XM2VTSDB: The Extended M2VTS Database,"Audio and Video-Based Biometric Person Authentication, pp. 72-77, 1999.

M. Choras, "Ear Biometrics Based on Geometrical Feature Extraction," Electronic Letters on Computer Vision and Image Analysis, vol. 5, pp. 84-95, 2005. 
M. Choras, "Further Developments in Geometrical Algorithms for Ear Biometrics," Proc. Fourth Int'l Conf Articulated Motion and Deformable Objects, pp. 58-67, 2006.

B. Luo, A.D. Cross, E.R. Hancock, "Corner detection via topographic analysis of vector potential", Pattern Recognition Letter. 20 (6) (1999) 635-650.

N. Ahuja, "A transform for multiscale image segmentation by integrated edge and region detection", IEEE Trans. PAMI 18 (12) (1996) 1211-1235.

N. Ahuja, J.H. Chuang, Shape representation using a generalized potential field model, IEEE Trans. PAMI 19 (2) (1997) 169-176.

Li Yuan, Zhichun $\mathrm{Mu}$, Zhengguang $\mathrm{Xu}$, "Using Ear Biometrics for Personal Recognition”, IWBRS 2005 , Beijing, China, October 2005, 221-228.
C. Xu, J.L. Prince, "Gradient vector flow: a new external force for snakes", in: Proc. IEEE Conf. on Computer Vision and Pattern Recognition (CVPR), 1997, pp. 66-71.

R. M. Haralick. L. T. Watson, and T. J. Laffey, "The topographic primal sketch," Int. J . Robotics Res. vol. 2, pp. 50-72, 1983.

J. Wang, and L. Yin, "Static topographic modeling for facial expression recognition and analysis," Computer Vision and Image Understanding Journal, vol. 108, pp. 1934, October 2007.

L. Wang, and T. Pavlidis."Direct Gray-Scale Extraction of Features for Character Recognition",IEEE Trans. PAMI. vol. 15, no. 10, pp.1053-1067, October 1993.
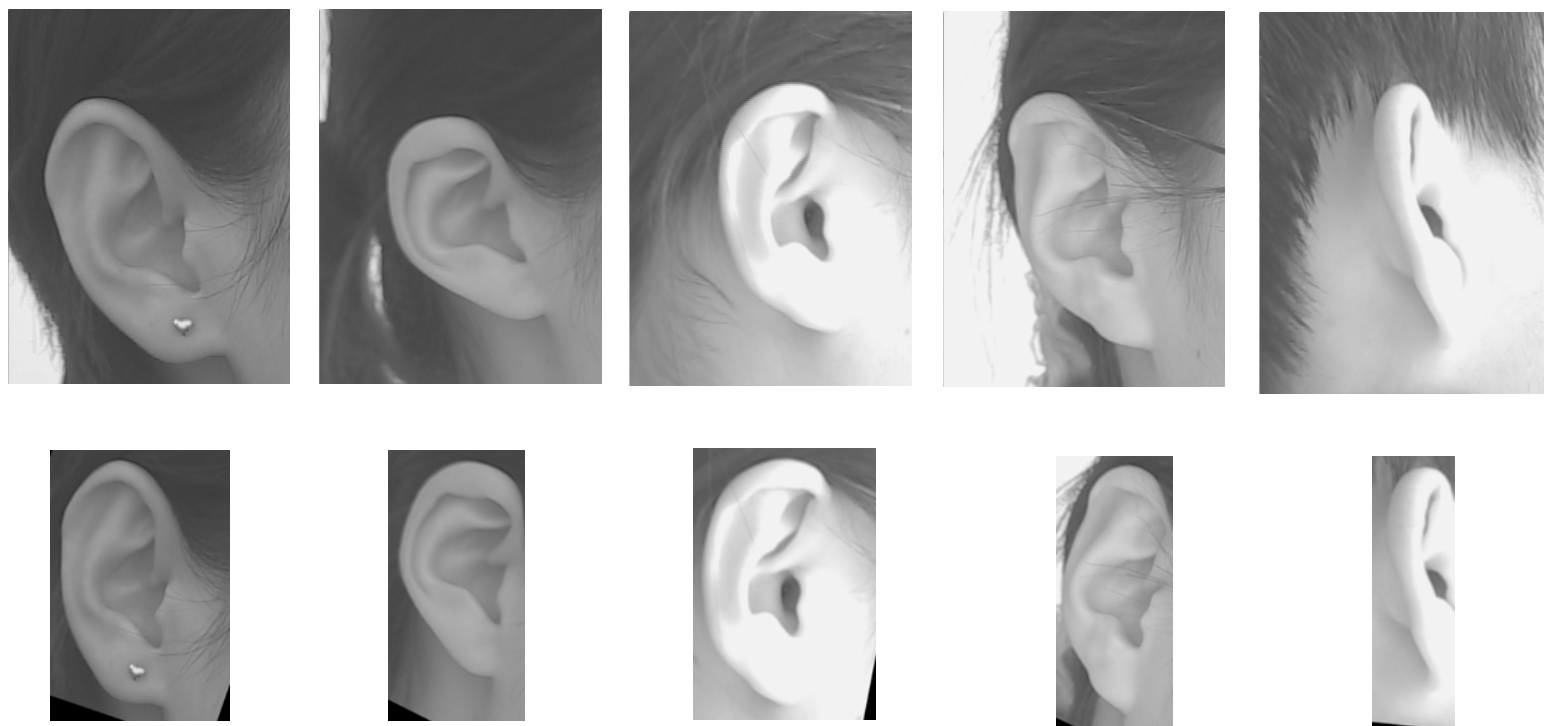
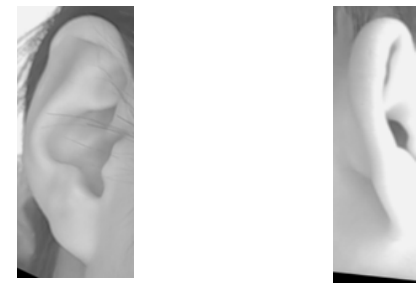

Figure10: A. Some samples from ear images in USTB database (first row), B. The results of segmentation of the first row using the proposed algorithm (second row). 